\title{
Comparison Between Digital and Ordinary Methods in Analyzing Cephalometric Radiographic Images
}

\author{
Mehrdad Panj Noush ${ }^{1}$ and Masomeh Esmaily ${ }^{2, *}$ \\ ${ }^{1}$ Department of Radiology, Tehran University of Medical Sciences, Tehran, IR Iran \\ ${ }^{2}$ Department of Orthodontics, Lorestan University of Medical Sciences, Khorramabad, IR Iran \\ *Corresponding author: Masomeh Esmaily, Department of Orthodontics, Lorestan University of Medical Sciences, Khorramabad, IR Iran. Tel: +98-9126879428, Fax: +98-2122039033, \\ E-mail:dr.esmaily@yahoo.com
}

Received 2014 November 2; Accepted 2014 December 9.

\begin{abstract}
Background:Diagnosis and treatment planning in orthodontic and orthognathic surgery is greatly related to the results of cephalometric analysis report. Manual analysis is time taking and if the precision and accuracy of this technique can be achieved by digital method, time is saved considerably. On the other hand digital programs have fewer errors compared to manual methods done by human. Any mistake in the process of analysis can lead to wrong diagnosis and treatment planning and finally treatment failure.

Objectives: The main aim of this study was to compare the linear and angular measures between the two methods, digital and manual. No significant difference between these measures in the two methods will result in that digital method can be used instead of manual in order to save time.

Materials and Methods: This study was conducted on 40 cephalometric images taken from a radiology clinic. The samples were analyzed by computer after determining key points. Romexis ${ }^{\circledR}$ (planmeca Romexis ${ }^{\circledR}$ cephalometric analysis) software was used for computer analysis. The samples were then printed and analyzed manually. At last all the measures obtained from 11 different landmarks by two observers were compared by interclass correlation coefficients (ICC) for reliability.

Results: The results of this study showed that the obtained data was 100 percent reliable. On the other hand no significant difference was observed in each of the evaluated landmarks between two methods.

Conclusions: It seems that cephalometric analysis software is not only reliable but also saves time and reduces manual analysis errors.

Keywords: Cephalometric Analysis Software, Cephalometric Radiography, Manual Analysis
\end{abstract}

\section{Background}

Diagnosis and treatment planning in orthodontic treatment and orthognathic surgery is largely dependent on the results of cephalometric analysis. Orthodontist should evaluate different aspects of functional, physical and behavioral characteristics of the patient such as psychosomatic problems, dentoalveolar disturbances (1), physiologic effect of malocclusion on movement of lips and jaw, respiration, growth, mastication, speech, the health of oral cavity, anatomic aspects of malocclusion, occlusion, tooth and jaw relations, form of face, asymmetry of face and dental system. One of the main important concepts in orthodontics is determining the relation between skeletal compartments especially those parts which are related to each other and to the skull. Cephalometric analysis can be helpful in this regard although the interpretation of data still is on debate $(2,3)$. Since the introduction of lateral cephalometry in 1931 by Broadbent this type of radiography is routinely used for diagnosis and treatment planning or follow up the patients after treatment. The decisions made by orthodontist are all based on this type of radiograph (4). The most common method for tracing and analysis is the one made by hand. This technique takes a lot of time (5) and measurement mistakes can be expected (6). The most common mistakes happen during finding anatomic landmarks $(7,8)$. This is related to many different factors such as knowledge and experience of practitioner, visual acuity, and quality of radiograph (9). Other mistakes happen when exposing the film and in the processing step and these mistakes refer back to equipment or technique problems. Cephalometric analysis can be done by computer for this purpose. In this technique, landmarks are defined by hand and then analyzed by computer. Computer analysis can reduce the number of mistakes that may be done in manual methods. These mistakes can happen when drawing lines by a ruler or measuring an angle by conveyor (5). On the other hand, when landmarks are located by hand, mistakes maybe happened when locating them, but finally the amount of mistakes in the computer based method will not be more than manual method (10). At the same time some studies have shown that there is significant difference between the results obtained from manual method

Copyright (C 2015, Iranian Journal of Orthodontics. This is an open-access article distributed under the terms of the Creative Commons Attribution-NonCommercial 4.0 International License (http://creativecommons.org/licenses/by-nc/4.0/) which permits copy and redistribute the material just in noncommercial usages, provided the original work is properly cited. 
and those from computer based methods (11). Recent studies show that although the difference between the results of data analysis from original radiograph compared with digital copy may be significantly different, there is no clinical difference (12). The characteristics of digital radiography analysis are fast measurements, easy treatment planning, reducing chemical substances and environmental pollution caused by the process of film development, easy data transfer, possibility of superimposing different films taken from the same patient and cheaper duplication. If the precision and accuracy of this method can be obtained by digital techniques, time can be saved. The function of digital programs may also reduce the number of errors made by manual methods. Several different computer programs have been designed to analyze cephalometric images. Studies were done to evaluate programs like Quick ceph, The Dolphin Imaging and Dentofacial Planner. The common result of these studies show that although there are differences between manual and computer based programs (which in some studies have shown to be statistically significant) the clinical outcome has been the same. The differences showed that some landmarks were more reliable in manual and some other more reliable in computer based method $(13,14)$. According to producing company (Planmeca), Romexis cephalometric analysis software has made it easier to analyze and superimpose cephalometric images for orthodontic treatment planning taking advantage of its proper tools. An actually easy to use and flexible program of this software enables the operator to easily analyze complex cases and to be practical at the same time.

\section{Objectives}

The main aim of this study was to compare linear and angular measurements between digital and manual methods.

\section{Materials and Methods}

40 digital cephalometric images were analyzed by two observers for 2 times using Romexis software on a Chinese 15.4 Inch LCD monitor (Dell Vostro 1520). The images were collected from the archive of a private oral radiology clinic. Images were printed and analyzed manually using conveyor on a LED view box. In this analysis 15 anatomic landmarks and 11 linear angular variants were located by two observers twice to find out the reliability. ICC (interclass correlation coefficient) was used to compare reliability.

\subsection{Lateral Cephalometric Tracing Landmarks}

ANS: anterior nasal spine, the anterior tip of the nasal spine at the lower margin of the anterior nasal opening Ar: articulate: intersection of posterior ramus and inferior cranial base

Ba: Basion, the most inferior part on the anterior edge of foramen magnum
Co: condylion, The most posterior-superior point on the curvature of the condylar head.

Go: Gonion, the most inferior, posterior and lateral point on the external angle of the mandible

Gn: Gnathion, the most inferior midline point on the mandible porion

Me: Menton, the most inferior point of the chin on the outline of the symphysis

$\mathrm{N}$ : Nasion, the most anterior point of the nasofrontal suture

Or: Orbitale, the lowest point on the external border of the orbital cavity

PNS: posterior nasal spine, the most posterior point at the sagittal plane on the bony hard palate

Pog: pogonion, the most anterior point on the symphysis Po: Porion, the most superior point on the external auditory meatus

Point A: the innermost point on the contour of the maxilla between anterior nasal spine and the incisor

Point B: The most posterior point in the concavity along the anterior border of the symphysis

PTM: Pterygomaxillare, pterygomaxillary fissure is made by anterior tuberosity and pterygoid process of sphenoid bone.

$\mathrm{S}$ : Sella, is the centre of sella turcica

\subsection{Lateral Cephalometric Variables}

SNA: The angle between SN and NA

SNB: The angle between SN and NB

ANB: difference between SNA and SNB

IIA: Interincisal angle, angle between the maxillary and mandibular incisor axis

IMPA: angle formed by the intersection of the mandibular incisor axis to mandibular plane

NLA: angle formed between columella, subnasale, and the upper lip

\section{Results}

Data from this study showed that, as there was no significant difference between each of evaluated landmarks in computer and manual analysis. Table 1 summarizes data from the study. This table shows that the numbers of cases are 40 cephalometric images in each group. In each images 11 variants have been located. The variants are composed of dental and skeletal.

In Table 2 the reliability of data from both methods is discussed. Each of the variants was assessed individually and as a pair using ICC scale. Results show that data were completely reliable as all the variants showed 100\% reliability.

In Table 3, the minimum degree of difference shows the highest point in which computer based results are less than manual one and the maximum degree of difference is the highest point in which computer based results are higher than manual one. It is concluded from this table that in this research degree of errors has been least. 
Panj Noush M et al.

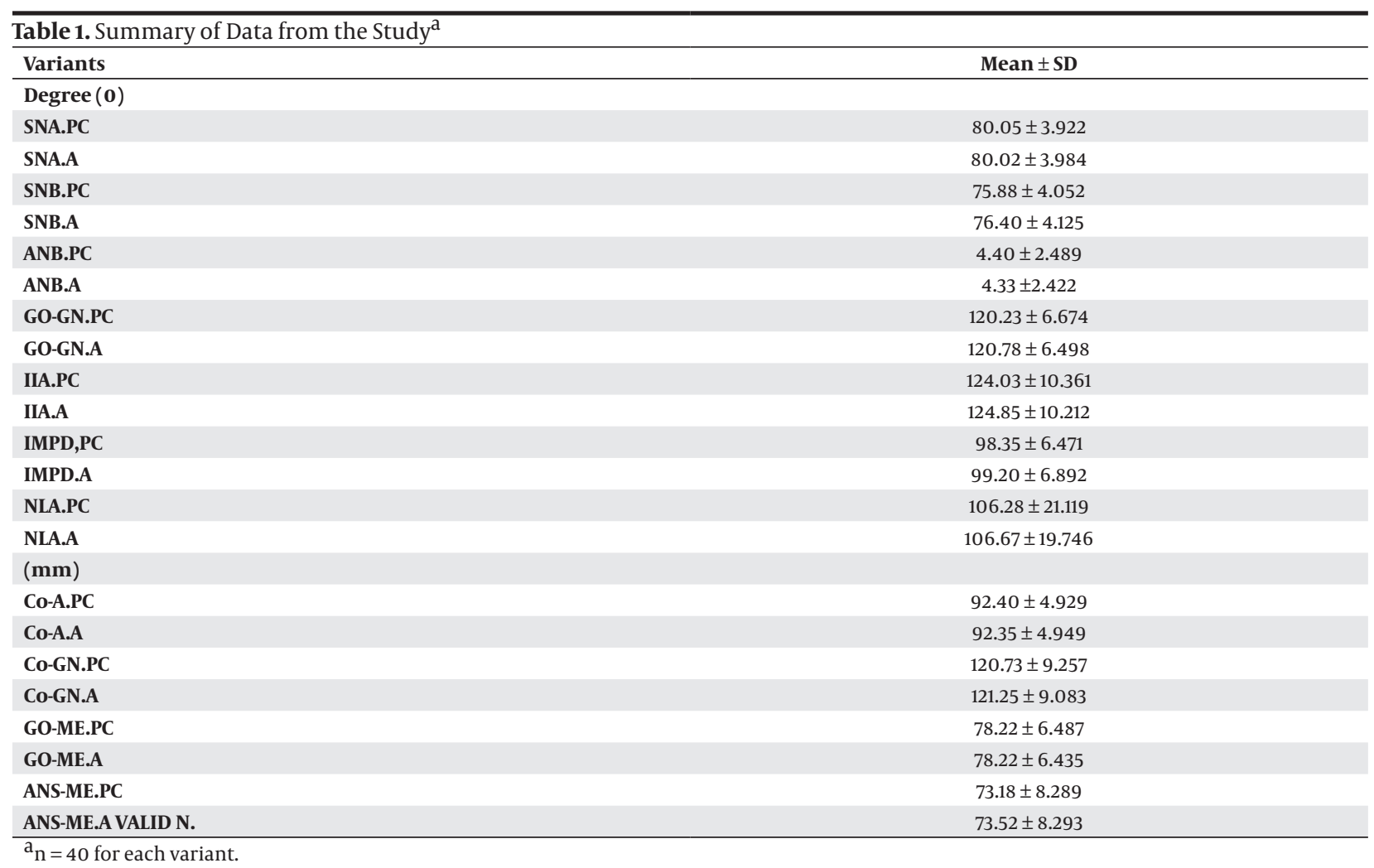

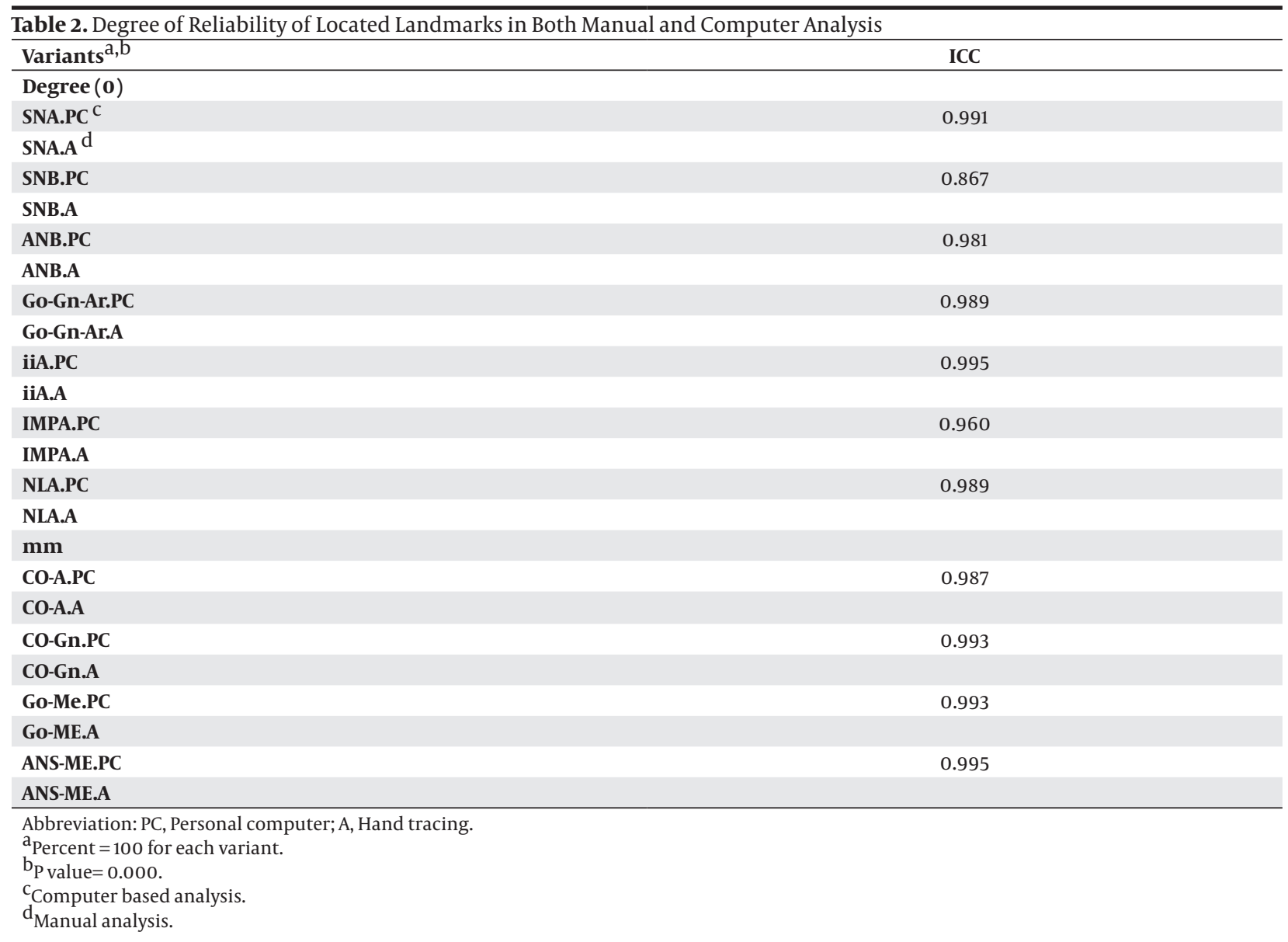


Panj Noush M et al.

\begin{tabular}{|c|c|c|c|}
\hline Variants $^{\mathrm{a}}$ & Minimum & Maximum & Mean \pm SD \\
\hline SNA.diff ${ }^{b}$ & -2 & 1 & $0.025 \pm 0.530$ \\
\hline SNA.dif.abs ${ }^{c}$ & 0 & 2 & $0.225 \pm 0.479$ \\
\hline SNB.diff & -10 & 2 & $-0.525 \pm 2.11$ \\
\hline SNB.d.abs & 0 & 10 & $0.825 \pm 2.01$ \\
\hline ANB.diff & -1 & 1 & $0.75 \pm 0.474$ \\
\hline ANB.d.abs & 0 & 1 & $0.225 \pm .0 .422$ \\
\hline Go-Gn-Ar.diff & -2 & 2 & $-0.55 \pm 0.985$ \\
\hline Go-Gn-Ar.d.abs & 0 & 2 & $0.85 \pm 0.735$ \\
\hline IIA.diff & -3 & 1 & $-0.825 \pm 1$ \\
\hline IIA.d.abs & 0 & 3 & $1.075 \pm 0.729$ \\
\hline IMPA.diff & -8 & 2 & $-0.85 \pm 1.88$ \\
\hline IMPA.d.abs & 0 & 8 & $1.2 \pm 1.68$ \\
\hline NLA.diff & -7 & 1 & $0-.4 \pm 2.98$ \\
\hline NLA.abs.d & 0 & 1 & $2.1 \pm 2.13$ \\
\hline Co-A.diff & -2 & 2 & $0.05 \pm 0.782$ \\
\hline Co-A.d.abs & 0 & 2 & $0.5 \pm 0.599$ \\
\hline Co-Gn.diff & -4 & 1 & $-.525 \pm 1.08$ \\
\hline Co-Gn.d.abs & 0 & 4 & $.725 \pm 0.960$ \\
\hline Go-Me.diff & -1 & 1 & $0.00 \pm 0.751$ \\
\hline Go-Me.abs & 0 & 1 & $.55 \pm 0.503$ \\
\hline ANS-Me.diff & -4 & 1 & $-0.35 \pm 0.833$ \\
\hline ANS-Me.d.abs & 0 & 4 & $0.5 \pm 0.751$ \\
\hline
\end{tabular}

$a_{n}=40$ for each variant.

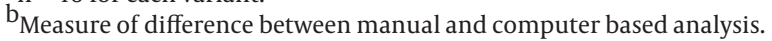

$\mathrm{c}_{\text {Absolute measure of errors. }}$

\section{Discussion}

This study was done on 40 cephalometric images using Romexis software by 2 observers on 15 anatomic landmarks and 11 linear angular variant. According to the results, there was no significant difference between any of the studied variants in both methods Sayinsu et al. (12) compared classic method of tracing with computer-based method. They scanned lateral cephalometric images with resolution of 300 dpi and made it digitalized in computer. In this study 2 operators analyzed the images twice, once by Dolphin Imaging software 9.0 and manually for the second time. Both methods were done by the same operators. The results showed that computer based method for tracing and analysis had no advantage over manual method considering accuracy. In another study, Dvortsin et al. (15) compared differences between manual tracing and tracing on monitor using soft tissue software analysis program. Twenty random cephalometric images with natural head position were selected. A computer program named View box 3.1.1.9 cephalometric software was used. The second image was prepared one week after the first one. The results showed that the difference was mainly in stomion landmark. It

means that tracing has been different in this zone. Different position of lips when taking the images was regarded to be the reason for the difference. This landmark is one of the most difficult points to be marked on the radiographic image. Other points had a difference less than one degree and $1 \mathrm{~mm}$. according to the results of this study significant difference $(\mathrm{P}<0.005)$ was found between tracing by computer and manually using soft tissue software. Naoumova et al. (16) compared manual and digital tracing of scanned radiographic images using FACAD software. The images were taken from 30 patients ( 12 males and 18 females with mean age of 25) before and after maxillofacial surgery. A practitioner identified 25 landmarks once by a computer software and once manually. T test was used to analyze the data. The results showed that both methods had approximately the same results. No significant difference was seen between most of the landmarks that once were analyzed by software and once manually. Some landmarks like Gn and Si showed significant statistical difference comparing 2 methods. At the same time no clinical difference was noted to happen eventually. Celik (17) used different methods 
in his study. The images were prepared by digital method at first. After analyzing by JOE and Vistadent 2.1AT, the images were printed and analyzed manually. The results showed that different landmarks might show different results. Nasolabial angle showed unreliable results in all the three methods. This shows that this point cannot be identified exactly and it needs to be identified with great care. Singh et al. (18) compared cephalometric tracing between PACS (picture archiving and communication system) and manual method .PACS is a managing medical information system, which is used for archiving and measuring dental and medical images. This software is not specific for lateral cephalograms so the aim of the study was to evaluate its efficiency for cephalometric radiograph tracing. Six angles and 4 linear parameters were evaluated in this study. Five radiographs were selected for this study (4 females and 1 male between 14 to 20 years old). The results showed that only SNB and lower incisal angle showed significant difference with only minor discrepancies. In other landmarks no significant difference was observed. The study points out that PACS can be used in measuring and tracing cephalometric landmarks. Uysal et al. (4) in a similar study to ours compared manual and computer based analysis methods and evaluated the reliability of landmarks under study. After evaluating 100 cephalometric images, the author states that there is no considerable difference between the 2 methods but the average time spent to trace the points in manual method was near 4 minutes more than computer based analysis. The study mentions that none of the methods was more reliable than other. However, increasing the speed of analysis can be an advantage of computer tracing. Uysal used Dolphin software in this study which is a wellknown software for cephalometric analysis. Sayinsu (12) reported that there is not great advantage for manual or computer based analysis for tracing cephalometric images. He used Dolphin software in his study. He also points out that scanner and monitor resolution can be of importance in reporting the results. As an example he used scanner with 300 dpi resolution for his study. It seems that this resolution which was also used in our study is a proper one for evaluating digital radiographic images. The overall results indicate that different software and different locations can present different statistical results but this variation has no clinical effect and in both conditions, the clinical outcome will be the same.

\subsection{Conclusion}

it can be concluded that in both methods whether manual or computer based, the clinical results are the same. What that is important for both patient and orthodontist is the clinical aspect of the tracing. Using software can increase the speed and save time used for analysis. It is also mentioned that some common mistakes in manual tracing like mistakes made when measuring angles or drawing lines do not happen in computer based analysis. It is recommended to make use of computer based analysis in challenging cases such as soft tissue analysis and important cases like before orthognathic surgery. It is also recommended to perform more studies in the future especially on such cases.

\section{References}

1. Fleischer-Peters A, Scholz U. [Orofacial dyskinesias from the psychosomatic viewpoint]. Fortschr Kieferorthop. 1985;46(3):181-90. [PubMed:3860460]

2. Brown M. Eight methods of analysing a cephalogram to establish anteroposterior skeletal discrepancy. Br J Orthod.1981;8(3):139-46. [PubMed: 7020748]

3. Hocevar RA, Stewart MC. A study of reference lines for mandibular plane angles. American Journal of Orthodontics and Dentofacial Orthopedics. 1992;102(6):519-26. [PubMed:1456232]

4. Uysal T, Baysal A, Yagci A. Evaluation of speed, repeatability, and reproducibility of digital radiography with manual versus computer-assisted cephalometric analyses. Eur J Orthod. 2009;31(5):523-8. doi: 10.1093/ejo/cjp022. [PubMed: 19443692]

5. Liu JK, Chen YT, Cheng KS. Accuracy of computerized automatic identification of cephalometric landmarks. American Journal of Orthodontics and Dentofacial Orthopedics. 2000;118(5):535-40. [PubMed: 11094367]

6. Chen YJ, Chen SK, Chung-Chen Yao J, Chang HF. The effects of differences in landmark identification on the cephalometric measurements in traditional versus digitized cephalometry. The Angle orthodontist. 2004;74(2):155-61. [PubMed:15132440]

7. Houston WJB. The analysis of errors in orthodontic measurements. American journal of orthodontics. 1983;83(5):382-90. [PubMed: 6573846]

8. Lindsten R, Ögaard B, Larsson E. Difference in dental lateral arch length between 9-year-olds born in the 1960s and the 1980s. American Journal of Orthodontics and Dentofacial Orthopedics. 2000;117(6):663-8. [PubMed:10842109]

9. Ongkosuwito EM, Katsaros C, van 't Hof MA, Bodegom JC, Kuijpers-Jagtman AM. The reproducibility of cephalometric measurements: a comparison of analogue and digital methods. Eur J Orthod. 2002;24(6):655-65. [PubMed:12512783]

10. Gravely JF, Benzies PM. The clinical significance of tracing error in cephalometry. British Journal of Orthodontics. 1974;1(3):95-101. [PubMed: 4525738]

11. Chen YJ, Chen SK, Huang HW, Yao CC, Chang HF. Reliability of landmark identification in cephalometric radiography acquired by a storage phosphor imaging system. Dentomaxillofac Radiol. 2004;33(5):301-6. doi:10.1259/dmfr/85147715. [PubMed:15585806]

12. Sayinsu K, Isik F, Trakyali G, Arun T. An evaluation of the errors in cephalometric measurements on scanned cephalometric images and conventional tracings. Eur J Orthod. 2007;29(1):105-8. doi:10.1093/ejo/cjlo65. [PubMed:17290023]

13. Gerbo LR, Poulton DR, Covell DA, Russell CA. A comparison of a computer-based orthognathic surgery prediction system to postsurgical results. The International journal of adult orthodontics and orthognathic surgery. 1996;12(1):55-63. [PubMed: 9456618]

14. Turner PJ, Weerakone S. An evaluation of the reproducibility of landmark identification using scanned cephalometric images. J Orthod. 2001;28(3):221-9. doi: 10.1093/ortho/28.3.221. [PubMed: 11504900]

15. Dvortsin DP, Sandham A, Pruim GJ, Dijkstra PU. A comparison of the reproducibility of manual tracing and on-screen digitization for cephalometric profile variables. The European Journal of Orthodontics. 2008;30(6):586-91. [PubMed: 18719051]

16. Naoumova J, Lindman R. A comparison of manual traced images and corresponding scanned radiographs digitally traced. Eur J Orthod. 2009;31(3):247-53. doi: 10.1093/ejo/cjn110. [PubMed: 19342425]

17. Celik E, Polat-Ozsoy O, Memikoglu TUT. Comparison of cephalometric measurements with digital versus conventional cephalometric analysis. The European Journal of Orthodontics. 2009:cjn105.

18. Singh P, Davies TI. A comparison of cephalometric measurements: a picture archiving and communication system versus the hand-tracing method-a preliminary study. The European Journal of Orthodontics. 2011;33(4):350-3. [PubMed: 20923935] 